\title{
A new method for improving LC/Time-of- flight mass spectrometry detection limits using simultaneous ion counting and waveform averaging
}

Yosuke Kawai ${ }^{\star a}$, b, Yumi Miyakeb, Toshinobu Hondob,c, Jean-Luc Lehmannd, Kentaro Teradaa,b, Michisato Toyodab

aDepartment of Earth and Space Science, Graduate School of Science, Osaka University, Osaka, 1-1

Machikaneyama, Toyonaka, Osaka 560-0043, Japan

bProject Research Center for Fundamental Sciences, Graduate School of Science, Osaka University, Osaka, 1-1 Machikaneyama, Toyonaka, Osaka 560-0043, Japan

cMS-Cheminformatics Limited Liability Corporation, 2-13-21 Sasaonishi, Toin, Mie 511-0231, Japan

dAcqiris SA, Chemin des Aulx 12, 1228 Plan-les-Ouates, Switzerland

\section{Corresponding Author}

*Yosuke Kawai - Department of Earth and Space Science, Graduate School of Science, Osaka University, 1-1 Machikaneyama, Toyonaka, Osaka 560-0043, Japan; orcid.org/0000-0002-4166-7878 Email: ykawai@ess.sci.osaka-u.ac.jp 


\section{Supplemental Information}

$\underline{\text { Supplemental Figures }}$

Supplemental Figure S1: The frequency histogram of the single ion peak height at various detector voltage.

Supplemental Figure S2: A schematic diagram of peak centroid calculation.

Supplemental Figure S3: The linear calibration curves of SDD and SDMX in the range from 0.02 to 0.2 pmol injected (Zoomed portion of Figure 4). 


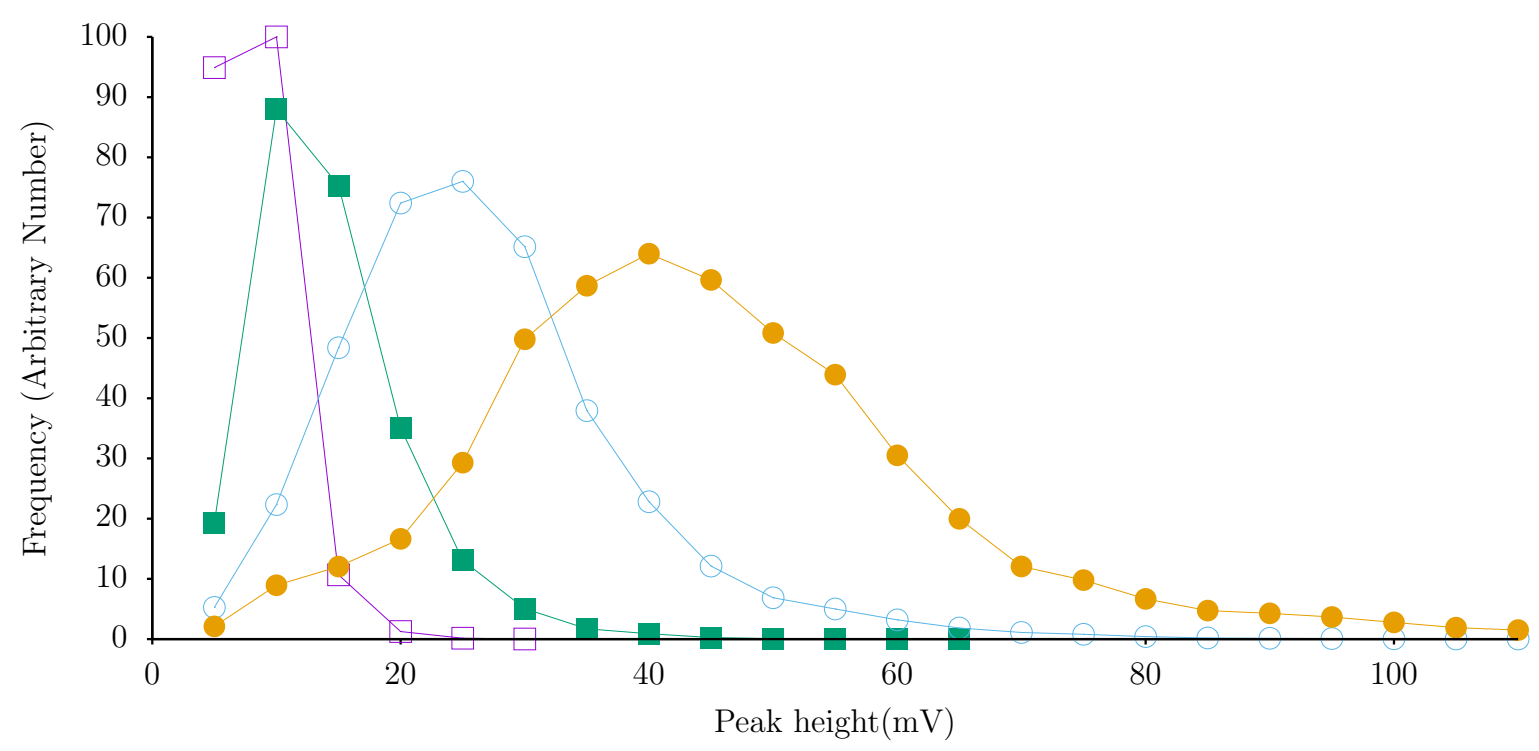

Supplemental Figure S1: The frequency histogram of the single ion peak height at various detector voltage. Detector voltages are, open square: $2000 \mathrm{~V}$, closed square: $2100 \mathrm{~V}$, open circle: $2200 \mathrm{~V}$, and closed circle $2300 \mathrm{~V}$. 


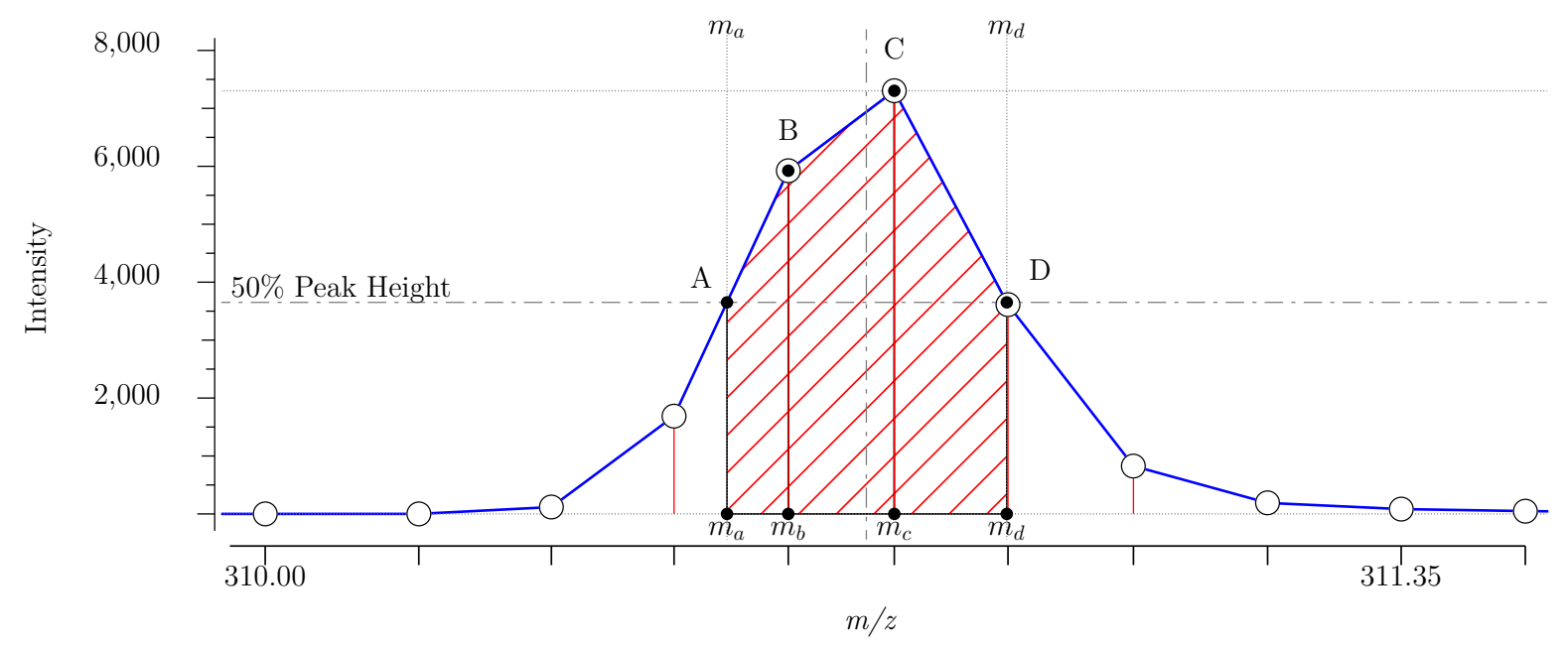

Supplemental Figure S2: A schematic diagram of peak centroid calculation. Peak centroid for each peak is determined by calculating the first moment of area from a trapezium series, where each trapezium consists of a sample point's intensity while the signal level is higher than $50 \%$ of peak height and the linear interpolated time point where the signal crosses the $50 \%$ threshold level. 

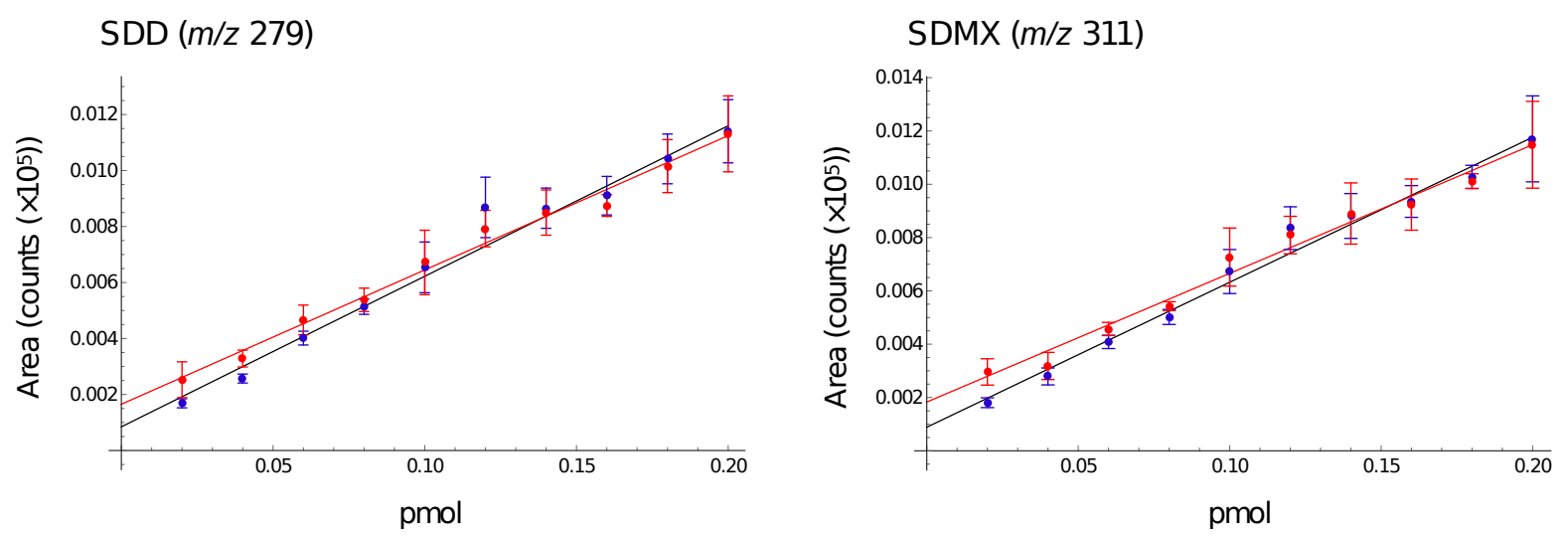

Supplemental Figure S3: The linear calibration curves of SDD and SDMX in the range from 0.02 to 0.2 pmol injected (Zoomed portion of Figure 4). All data points were collected with the PKD mode (blue). For comparison, the results of the AVG mode (red) are also plotted. The peak of the target compounds cannot be identified from the AVG waveform. So the area was calculated from the mass range determined by the PKD chromatogram. The intersection of the linear regression line for the PKD mode data analysis is closer to the origin compared to the AVG mode analysis. At low count rate, the peak area determination is affected by the noise signals for the AVG mode measurement. In contrast, with the PKD mode analysis, since the noise was not incorporated into the chromatogram, the peak area can be determined with high quantitative accuracy. 